\title{
Involvement of the mitochondrial pathway in bruceine D-induced apoptosis in Capan-2 human pancreatic adenocarcinoma cells
}

\author{
LING LIU ${ }^{1,2}$, ZHI-XIU LIN ${ }^{1}$, PO SING LEUNG ${ }^{2}$, LI-HUA CHEN ${ }^{2}$, MING ZHAO $^{1}$ and JUAN LIANG ${ }^{2}$ \\ ${ }^{1}$ School of Chinese Medicine, Faculty of Science, ${ }^{2}$ School of Biomedical Sciences, Faculty of Medicine, \\ The Chinese University of Hong Kong, Shatin, NT, Hong Kong SAR, P.R. China
}

Received November 16, 2011; Accepted January 2, 2012

DOI: $10.3892 / \mathrm{ijmm} .2012 .980$

\begin{abstract}
The fruit of Bruceajavanica L. is a common herbused in Chinese medicine for the treatment of a variety of cancers. Our research group has previously identified bruceine D (BD), a quassinoid found abundantly in $B$. javanica, to have potent cytotoxic effect on a number of pancreatic cancer cell lines, including Panc-1, SW1990 and Capan-1 cells. In the present study, we showed that BD was also able to inhibit the growth of the Capan-2 human pancreatic adenocarcinoma cell line, but it exerted only modest cytotoxicity on the WRL68 human hepatocyte cell line and a human pancreatic progenitor cell line. The antiproliferative effects of BD were comparable to those exhibited by camptothecin and gemcitabine in our culture system. We found a dose-dependent decrease of the mitochondrial membrane potential in BD-treated Capan-2 cells as measured by the JC-1 assay. BD exposure was able to attenuate the expression of Bcl-2 protein in Capan- 2 cells as detected by western blot analysis. In addition, the expression of both caspase 9 and caspase 3 in BD-treated Capan-2 cells was significantly accentuated. Moreover, BD was capable of inducing the fragmentation of genomic DNA in Capan-2 cells as evidenced by Hoechst staining. Cell cycle analysis demonstrated that $\mathrm{BD}$ could increase the percentage of Capan-2 cells in the subG $\mathrm{G}_{1}$ phase in a dose-related manner. An increase in the apoptosis of Capan-2 cells was also observed by Annexin V and PI staining. These results unequivocally indicate that $\mathrm{BD}$ induces cytotoxicity in Capan-2 cells via the induction of cellular apoptosis involving the mitochondrial pathway.
\end{abstract}

Correspondence to: Professor Zhi-Xiu Lin, School of Chinese Medicine, Faculty of Science, The Chinese University of Hong Kong, 1/F., Sino Building, Shatin, NT, Hong Kong SAR, P.R. China

E-mail:1inzx@cuhk.edu.hk

Professor Po Sing Leung, School of Biomedical Sciences, Faculty of Medicine, The Chinese University of Hong Kong, Shatin, NT, Hong Kong SAR, P.R. China

E-mail:psleung@cuhk.edu.hk

Key words: apoptosis, bruceine D, pancreatic cancer, Capan-2 cells, mitochondrial pathway

\section{Introduction}

Human pancreatic cancer is a gastrointestinal malignancy with extremely poor prognosis owing to a lack of early clinical diagnosis and its intrinsic resistance to radiotherapy and chemotherapy $(1,2)$. According to the Hong Kong Cancer Registry in 2009 pancreatic cancer was the 8th and 6th major cause of cancer-related deaths in males and females, respectively. As for the management of pancreatic cancer, surgical resection remains the only potentially curative treatment of pancreatic cancer. However, only 5-25\% of the patients diagnosed with pancreatic cancer were operable, and chemotherapy thus remains the mainstay of palliative management for the locally advanced and metastatic stage of this cancer. The median disease-free survival following complete resection of the pancreatic tumor and adjuvant chemotherapy with the first line chemotherapeutic agent gemcitabine is 13.4 and 6.9 months, respectively (3-6). Although camptothecin has a positive effect on arresting pancreatic tumor growth, many unwanted sideeffects limit its clinical application (5,7). All these dismal data highlight an urgent need for new therapeutic agents that could produce better clinical outcomes for this deadly disease.

Our previous studies have shown that the fruit of Brucea javanica L., a Chinese medicinal plant commonly used for cancer treatment, has potent in vitro anti-pancreatic cancer activity (8). Furthermore, bruceine D (BD), a quassinoid found in abundance in $B$. javanica, was identified as the principal active ingredient of this cancer-treating Chinese herb (9). In order to evaluate the potential of BD as a chemotherapeutic agent for pancreatic cancer, in the present study we investigated the anti-pancreatic cancer activity of BD on human pancreatic adenocarcinoma Capan-2 cells and elucidated the underlying mechanisms of action. In addition, the cytotoxic selectivity of BD on different cell lines was also determined.

\section{Materials and methods}

Chemicals. BD was purified from the fruit of Brucea javanica in our laboratory and its identity was confirmed based on nuclear magnetic resonance (NMR) and mass spectrometry (MS) data. The purity of BD, which was in white powder form, was $>95 \%$ based on high performance liquid chromatography (HPLC) analysis. The chemical structure of BD is shown in Fig. 1. 
General cell culture. Capan-2, a human pancreatic adenocarcinoma cell line and WRL68, a human hepatocyte cell line, were purchased from American Type Culture Collection (ATCC, VA, USA). For general cell culture, Capan-2 and WRL68 cells were routinely maintained in DMEM medium supplemented with $10 \%$ FBS, 4 mM L-glutamine, $4.5 \mathrm{~g} / 1$ glucose, $1.5 \mathrm{~g} / 1$ sodium bicarbonate, $100 \mathrm{U} / \mathrm{ml}$ penicillin and $10 \mathrm{U} / \mathrm{ml}$ streptomycin and incubated at $37^{\circ} \mathrm{C}$ in a $5 \% \mathrm{CO}_{2}, 95 \%$ air humidified atmosphere.

Human fetal pancreas tissue, obtained by extraction after termination of pregnancy between the 9th and 15th weeks of gestation, was provided by the Department of Obstetrics and Gynecology, Prince of Wales Hospital, The Chinese University of Hong Kong. Maternal consent and ethical approval from the Clinical Research Ethics Committee (CREC-2005.461) were obtained before the procedures. The experiments were performed using the preparations derived from a single fetal pancreas. To establish the primary culture of human pancreas, the fetal pancreas was minced and digested with $3 \mathrm{mg} / \mathrm{ml}$ collagenase P (Roche, Mannheim, Germany). Digested cell clusters were resuspended in modified RPMI-1640 medium supplemented with $10 \%$ FBS, $1 \mathrm{mM}$ sodium pyruvate, $1 \%$ penicillin and streptomycin, $10 \mathrm{mM}$ HEPES buffer (Invitrogen, Carlsbad, CA, USA) and 71.5 $\mu \mathrm{M} \beta$-mercaptoethanol (Sigma Aldrich, St. Louis, MO, USA) in $60 \mathrm{~mm}$ diameter non-treated culture dishes (Corning Incorporated, Lowell, MA, USA). Rounded, non-adherent cell clusters were formed within $48 \mathrm{~h}$ of incubation. Pancreatic progenitor cell (PPC) outgrowth from the cell clusters was induced by further addition of $20 \mathrm{ng}$ / $\mathrm{ml}$ each of basic fibroblast growth factor (Sigma Aldrich) and epidermal growth factor (Invitrogen) in the medium. A monolayer of PPCs from the cell clusters was established within 3-4 days, and the cells were harvested with $0.05 \%$ trypsin-EDTA (Invitrogen) upon reaching confluence.

Proliferation assay. The proliferation rates of Capan-2, WRL68 and PPC were determined by the SRB assay, a colorimetric end-point microplate assay which quantifies viable cells by staining their cellular protein content (10). BD was reconstituted in DMSO to produce a stock solution and then diluted with culture medium to various concentrations for cell culture experiments. As the first-line chemotherapeutic agent for locally advanced and metastatic pancreatic cancer, camptothecin (WAM, Osaka, Japan) and gemcitabine (Lilly France, Fegersheim, France) were included in the experiment as positive controls. Like BD, camptothecin was dissolved in DMSO to produce a stock solution. To minimize potential solvent effects on cell growth, the final concentration of DMSO in all culture wells were $<0.05 \%$. On the other hand, gemcitabine stock solution was prepared in PBS. Capan-2, WRL68 and PPC cells at $1 \times 10^{4}$ cells/well were incubated for $72 \mathrm{~h}$ with $\mathrm{BD}$, camptothecin and gemcitabine, respectively in 96-well plates.

After the incubation period, treatment-induced cytotoxicity was assessed by the SRB assay. Briefly, cells were fixed by addition of $25 \mu \mathrm{l}$ of cold $50 \%$ trichloroacetic acid (TCA, $4^{\circ} \mathrm{C}$ ) on the top of the $150 \mu 1$ of growth medium already present in each well. Each plate was incubated at $4^{\circ} \mathrm{C}$ for $1 \mathrm{~h}$. The medium and acid were then removed and the plate was washed four times with tap water to thoroughly remove the TCA, growth medium and dead cells. The remaining cells were allowed to

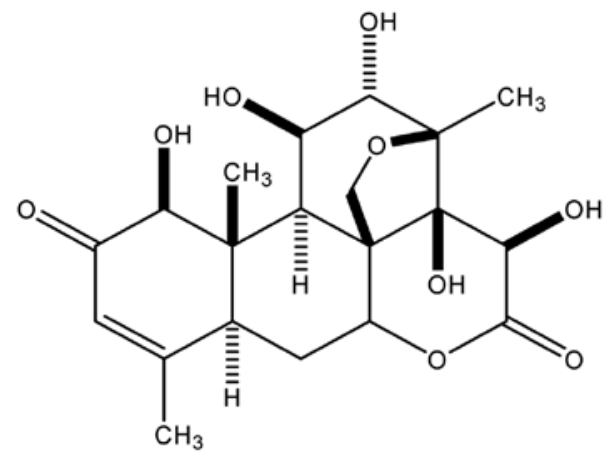

Figure 1. Chemical structure of brucein D.

air-dry at room temperature and then stained with $0.4 \%(\mathrm{w} / \mathrm{v})$ $\mathrm{SRB}$ at room temperature for $30 \mathrm{~min}$. The unbound dye was then removed with four washes in $1 \%$ acetic acid. Again, the plate was dried at room temperature or aided by a hair dryer. Bound SRB was dissolved in $150 \mu \mathrm{l}$ of $10 \mathrm{mM}$ Tris-base [tris(hydroxymethyl)amino methane] and the plate was shaken gently on a gyrator for $5 \mathrm{~min}$ before the optical density was measured at $550 \mathrm{~nm}$ using a microplate spectrophotometer (BioTek, Gene Co. Limited, VT, USA). The growth curves of different cell types were then constructed and the $\mathrm{IC}_{50}$ values were calculated using GraphPad Prism 5.01 computer software (GraphPad Software, Inc., San Diego, CA, USA).

Hoechst fluorescence staining for morphological evaluation. The DNA of the control and BD-treated cells was stained with Hoechst 33342 (Invitrogen). Approximately 3x10 Capan-2 cells/well were seeded in 6-well plates and incubated with $0,0.55,1.10$ or $2.20 \mu \mathrm{M} \mathrm{BD}$ for $32 \mathrm{~h}$, respectively. The cells were subsequently washed with PBS and fixed in $4 \%$ paraformaldehyde for $30 \mathrm{~min}$ at room temperature and then washed again with PBS. The fixed cells were incubated with $10 \mathrm{mg} / \mathrm{ml}$ Hoechst 33342 for $15 \mathrm{~min}$ at room temperature in the dark. Morphological changes of the BD-treated cells were examined using a fluorescence inverted microscope (IX-71, Olympus, Tokyo, Japan).

Cell cycle analysis with PI staining. Both adherent and floating cells were collected after $0.55,1.10$ and $2.20 \mathrm{mM}$ BD treatments for 24 and $48 \mathrm{~h}$. The collected cells were washed with ice-cold PBS followed by permeabilization with ice-cold $75 \%$ ethanol for at least $1 \mathrm{~h}$. The permeabilized cells $\left(10^{6}\right.$ cells $)$ were suspended in $0.5 \mathrm{ml} \mathrm{PI/RNase} \mathrm{solution} \mathrm{(BD} \mathrm{Pharmingen,} \mathrm{San}$ Diego, CA, USA) and incubated at room temperature for $15 \mathrm{~min}$ in the dark to apply the PI stain to cellular DNA. The stained cells were analyzed by a Cytomics FC500 flow cytometer (Beckman Coulter, Fullerton, CA, USA) with the fluorescence emission at $590 \mathrm{~nm}$ (FL3). DNA histograms were generated from the flow cytometry data and the percentage of cells in each cell cycle phase was determined. Cells with DNA contents less than that of the $G_{1}$ phase were considered apoptotic.

Apoptosis detection with Annexin $V$ and PI staining. Capan-2 cells with a density of $10^{6}$ cells/well were treated with $0.55,1.10$ and $2.20 \mathrm{mM} \mathrm{BD}$, respectively for $32 \mathrm{~h}$. The cells were collected and washed two times with ice-cold PBS and resuspended in 
binding buffer. After adjusting the cell density to $2-5 \times 10^{5}$ cells/ $\mathrm{ml}, 10 \mathrm{ml}$ of Annexin V-FITC (Invitrogen) was added to $190 \mathrm{ml}$ of cell suspension, followed by incubating the cells for $10 \mathrm{~min}$ at room temperature. Next, the cells were washed with $1 \mathrm{X}$ binding buffer and spinned down followed by resuspension in $190 \mathrm{ml}$ binding buffer. Ten microliters of $20 \mathrm{mg} / \mathrm{ml}$ propidium iodide stock solution was finally added to stain the cells. The stained cells were then analyzed by flow cytometry.

Estimation of the changes of mitochondrial membrane potential (MMP) by the JC-1 assay. Capan-2 cells were seeded at $0.3 \times 10^{5}$ cells/well in 6 -well plates and incubated with BD at $0.55,1.10$ and $2.20 \mu \mathrm{M}$ for $24 \mathrm{~h}$. The treated cells were collected and washed twice with ice-cold PBS, followed by addition of $10 \mathrm{ml}$ of $200 \mathrm{mM} \mathrm{JC}-1$ (Invitrogen) to each tube and incubation at $37^{\circ} \mathrm{C}, 5 \% \mathrm{CO}_{2}$ for $15-30 \mathrm{~min}$. The change of MMP was evaluated by flow cytometry at $488 \mathrm{~nm}$ excitation and $590 \mathrm{~nm}$ emission wavelengths.

Western blot analysis of Bcl-2, caspase 9 and caspase 3. Capan-2 cells at $2.2 \times 10^{6}$ cells/plate were seeded in $100-\mathrm{mm}$ plates. After 4-h incubation, the cells were exposed to BD at 2.20 and $11.0 \mu \mathrm{M}$ for $72 \mathrm{~h}$. The cells were then collected and lysed with CytoBluster protein extraction reagent (Novgen, Darmstadt, Germany). The protein concentrations were measured with the Micro BCA ${ }^{\mathrm{TM}}$ Protein Assay kit (Thermo Scientific, Rockford, IL, USA). Equal amount of proteins were then resolved by SDS-PAGE for $2 \mathrm{~h}$ at $100 \mathrm{~V}$ and transferred electrophoretically to PVDF membranes for $30 \mathrm{~min}$ at $15 \mathrm{~V}$. Membranes were blocked with $5 \%(\mathrm{w} / \mathrm{v})$ non-fat milk in PBS-T (0.1\% v/v Tween-20 in PBS) for $2 \mathrm{~h}$ and subsequently incubated overnight at $4{ }^{\circ} \mathrm{C}$ with primary anti-Bcl-2, primary anti-caspase 9, primary anti-caspase 3 and primary anti- $\beta$-actin antibody (Santa Cruz Biotechnology, Santa Cruz, CA, USA) at concentrations of mouse anti-Bcl-2 (1:1000), mouse anti-caspase 9 (1:1000), rabbit anti-caspase 3 (1:500) and mouse anti- $\beta$-actin $(1: 2000)$, respectively. The membrane was then incubated for $1 \mathrm{~h}$ at room temperature with HRP-conjugated sheep anti-mouse IgG (1:2000) and horseradish peroxidise (HRP)-conjugated donkey anti-rabbit IgG (1:1000), respectively. The protein bands were visualised by ECL western blotting detection reagents (Amersham Biosciences, Sweden). Rainbow molecular weight markers were used as size markers for the determination of protein size.

Statistical analysis. Multiple comparisons between data sets were performed using one-way analysis of variance (ANOVA) followed by the Dunnett's post-hoc test, in which the nontreated group served as the controls. Statistical analyses were conducted using a GraphPad Prism 5.01 software package (GraphPad Software, Inc.). A P-value of $<0.05$ was considered statistically significant.

\section{Results}

Effects of BD, camptothecin and gemcitabine on Capan-2 cell viability. As shown in Fig. $2 \mathrm{~A}$. both $\mathrm{BD}$ and camptothecin significantly inhibited the proliferation of Capan-2 cells, with $\mathrm{IC}_{50}$ values of 1.10 and $1.29 \mathrm{mM}$, respectively, as assessed by the SRB assay. As the first-line chemotherapeutic agent for locally advanced and metastatic pancreatic cancer, gemcitabine
A

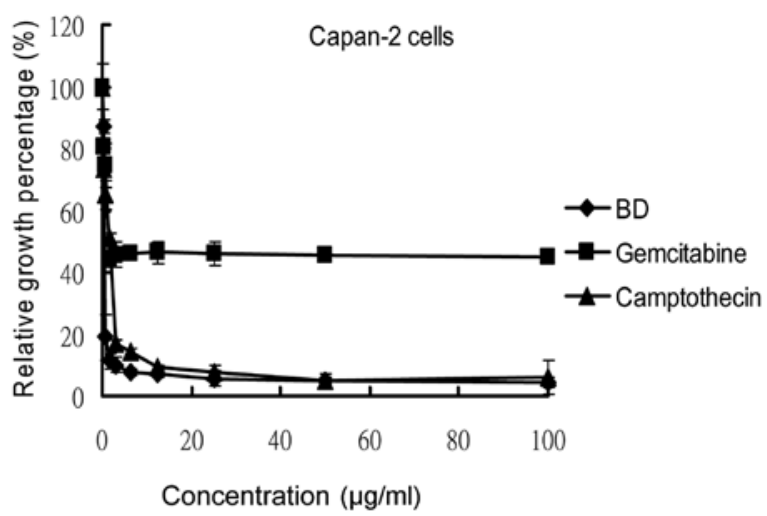

B

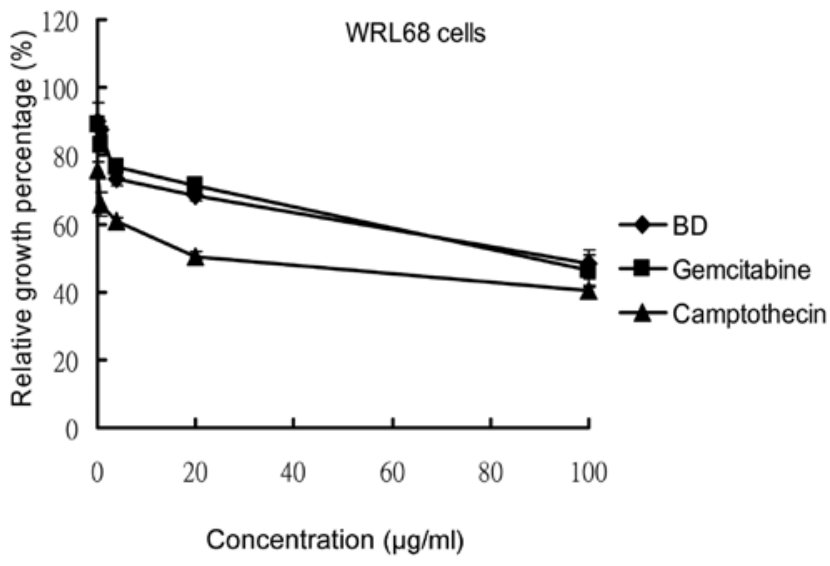

$\mathrm{C}$

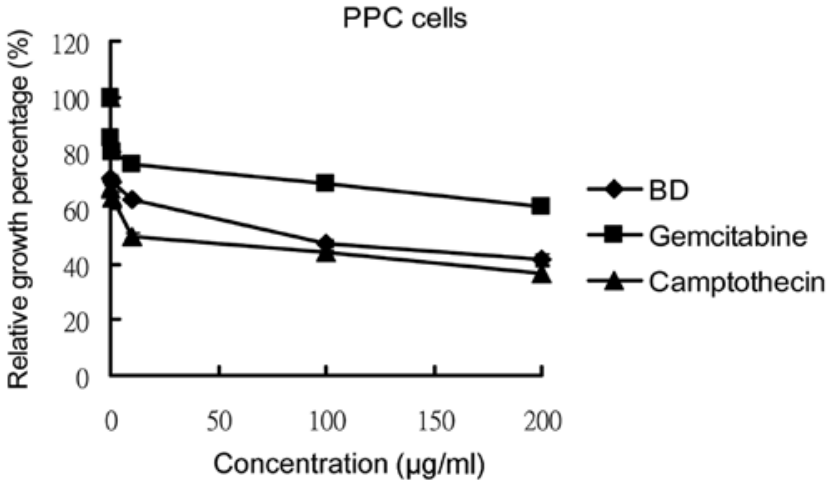

Figure 2. Effects of brucein D, gemcitabine and camptothecin on the viability of human pancreatic adenocarcinoma Capan-2 cells (A), human hepatocyte WRL68 (B) and human pancreatic progenitor cells PPC (C). Drug treatment was initiated $4 \mathrm{~h}$ after seeding the cells in 96-well plates. Cell viability was assessed by the SRB assay after $72 \mathrm{~h}$ of drug treatment.

exhibited a steady but modest cytotoxic effect on Capan-2 cells, with an $\mathrm{IC}_{50}$ value of $23.54 \mathrm{mM}$. On the other hand, BD showed a much less potent cytotoxic effect on cultured non-tumorigenic hepatocyte WRL68 and PPC cells, with $\mathrm{IC}_{50}$ values of 276.56 and $162.48 \mathrm{mM}$, respectively, indicating a very favorable cytotoxic profile of $\mathrm{BD}$ on pancreatic cancer. Table I summarizes the $\mathrm{IC}_{50}$ values of $\mathrm{BD}$, camptothecin and gemcitabine on different cell types. 

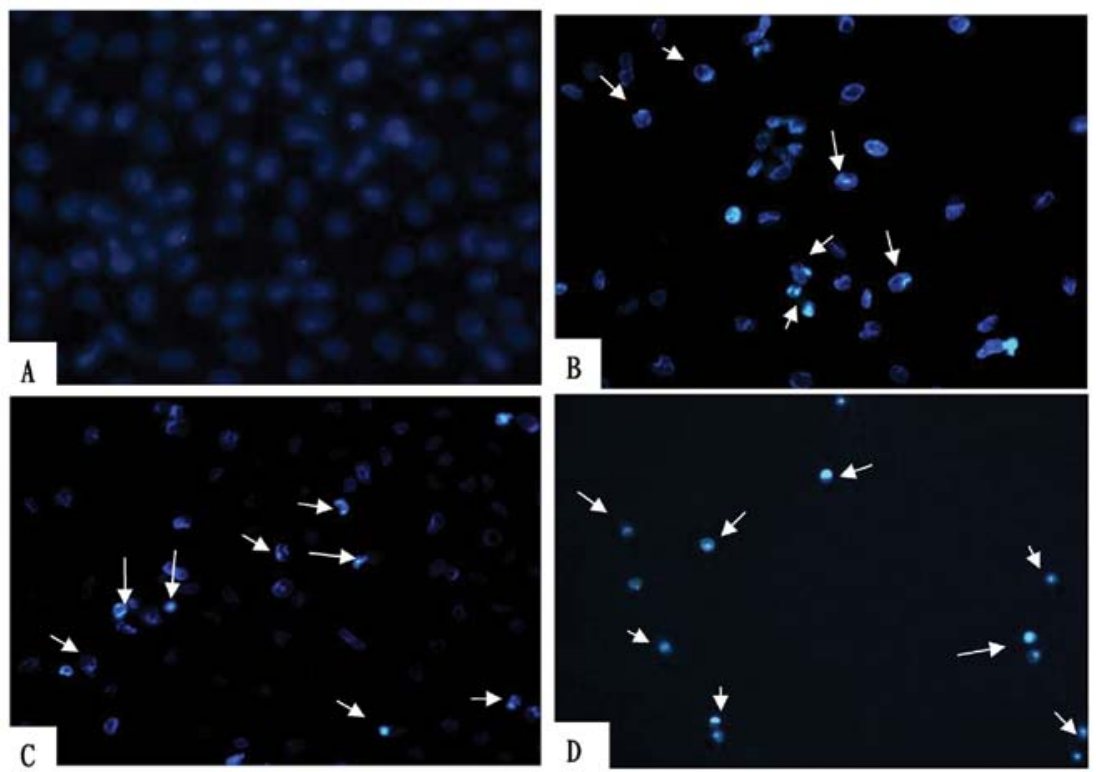

Figure 3. Morphological alteration in BD-treated Capan-2 as revealed by Hoechst 33342 fluorescence staining. Capan-2 cells were treated for $32 \mathrm{~h}$ with medium control (A); $0.55 \mu \mathrm{M} \mathrm{BD}$ (B); $1.1 \mu \mathrm{M} \mathrm{BD}$ (C); $2.2 \mu \mathrm{M} \mathrm{BD}$ (D). Note that nuclear condensation as indicated by arrows was observed in BD-treated cells.

Table I. $\mathrm{IC}_{50}$ values of Brucein D, gemcitabine and camptothecin of the growth of Capan-2, WRL68 and PPC cells.

\begin{tabular}{lrrr}
\hline & \multicolumn{3}{c}{$\mathrm{IC}_{50}(\mu \mathrm{M})$} \\
\cline { 2 - 4 } Cell line & Bruceine D & Gemcitabine & Camptothecin \\
\hline Capan-2 & 1.10 & 23.54 & 1.29 \\
WRL68 & 276.56 & 742.28 & 118.39 \\
PPC & 162.48 & $>759.87$ & 38.46 \\
\hline
\end{tabular}

"The $\mathrm{IC}_{50}$ values of the three compounds on the growth of Capan-2, WRL68 and PPC cells were determined by the SRB assay. ${ }^{* *} \mathrm{IC}_{50}$ values were calculated using a GraphPad Prism computer program.

Hoechst fluorescence staining for morphological changes of Capan-2 cells. Hoechst staining followed by observation under a fluorescence microscope revealed only slight Hoechst nuclear staining in non-treated (medium only) cells, and no apoptotic nuclei were observed (Fig. 3A). In contrast, 0.55 , 1.10 and $2.20 \mu \mathrm{M}$ BD treatment for $32 \mathrm{~h}$ dramatically altered Capan-2 cell morphology, in that Hoechst-stained Capan-2 cells displayed fewer intercellular connections and exhibited typical apoptotic morphology characterized by chromatin condensation and DNA fragmentation (Fig. 3B-D).

$B D$ on cell cycle progression. As shown in Fig. 4, flow cytometric analysis revealed that treatment with $\mathrm{BD}$ induced an increase in the percentage of subG ${ }_{1}$ phase (apoptotic cells) Capan-2 cells in a dose-dependent manner. When treated with $\mathrm{BD}$ at $0.55 \mu \mathrm{M}$ for $32 \mathrm{~h}$, the percentage of Capan-2 cells at subG $_{1}$ phase was only $4.9 \%$. When treated with $\mathrm{BD}$ at $1.10 \mu \mathrm{M}$ for $32 \mathrm{~h}$, the percentage of cells in the subG $\mathrm{G}_{1}$ phase was $19.8 \%$. An increase of BD to $2.20 \mathrm{mM}$ resulted in $26.0 \%$ of subG cells in the total cell population. In contrast, non-treated cells demonstrated normal cell viability with very few cells in the subG $\mathrm{G}_{1}$ phase.

Quantitation of apoptotic cells by Annexin V and PI staining. It has been observed that phosphatidylserine (PS) is translocated from the inner to the outer surface of the plasma membrane in the early stage of apoptosis. Therefore, PS exposure represents a useful target for evaluating apoptosis $(11,12)$. To assess plasma membrane changes, cells were stained with Annexin V (which binds preferentially to PS in the presence of $\mathrm{Ca}^{2+}$ ) and PI simultaneously. As shown in Fig. 5, the viable cells were localized in the lower left quadrant and the apoptotic cells were localized in the lower right quadrant. When treated with $\mathrm{BD}$ at $0,0.55,1.10,2.20 \mu \mathrm{M}$ for $32 \mathrm{~h}$, the percentages of apoptotic cells in the total cell population were, $0.1,2.3,7.7$ and $25.4 \%$, respectively, suggesting BD dose-dependently induced cellular apoptosis to Capan-2 cells.

$B D$-induced alteration of $M M P$ by the JC-1 assay. It is clear from Fig. 6 that MMP of Capan-2 cells was significantly attenuated by $\mathrm{BD}$ treatment. When treated with $\mathrm{BD}$ at $0,0.55,1.10$, $2.20 \mu \mathrm{M}$ for $24 \mathrm{~h}$, the number of cells with decreased MMP were $5.9,65.1,78.1$ and $86.7 \%$, respectively, indicative of the ability of BD to induce mitochondrial membrane depolarization, a distinct event leading to cellular apoptosis.

Western blot analysis. As shown in Fig. 7, exposure of Capan-2 cells to BD significantly attenuated the expression of Bcl-2 as evidenced by the appearance of a 27-30 kDa fragment (A). On the other hand, BD treatment markedly augmented caspase 9 and caspase 3 expression, as evidenced by the appearance of a $36-40 \mathrm{kDa}(\mathrm{B})$ and $19-21 \mathrm{kDa}(\mathrm{C})$ fragment respectively. These results unambiguously demonstrated that the underlying mechanism of BD-induced apoptosis in Capan-2 cells at the protein level involves the reduced expression of anti-apoptotic protein Bcl-2 and augmented activation of the pro-apoptotic proteins caspase 9 and caspase 3 . 

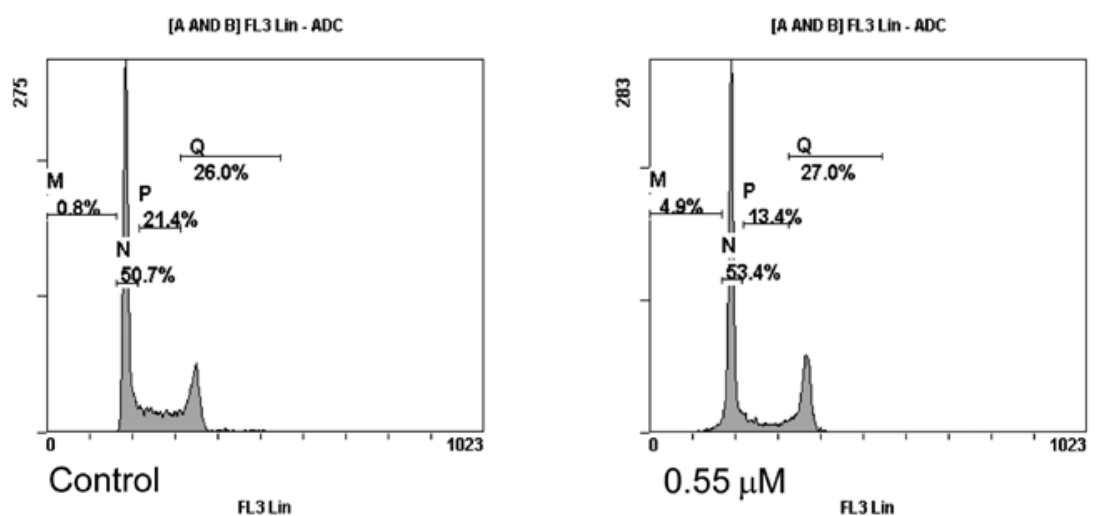

[A AND B] FL 3 Lin - ADC
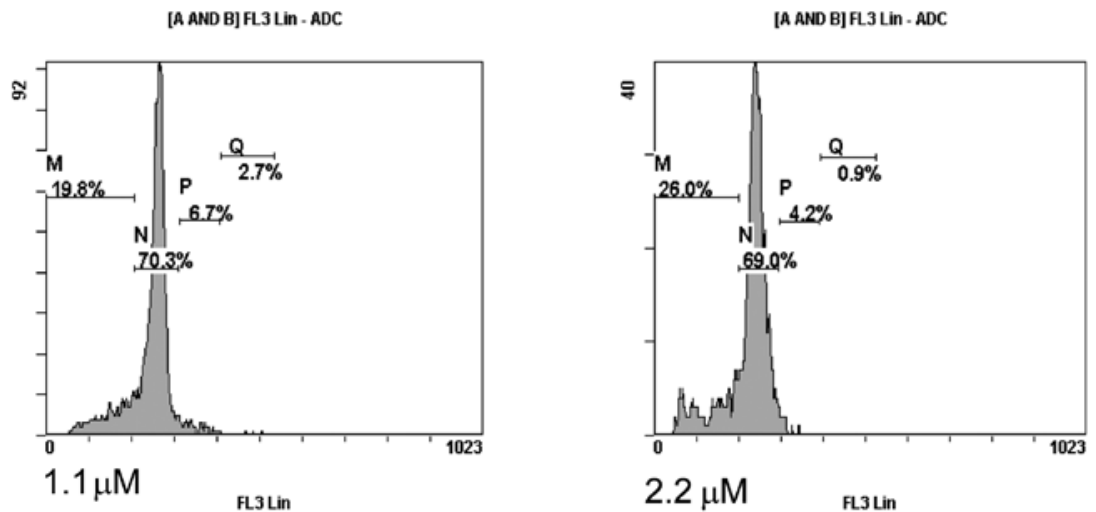

Figure 4. Cell cycle analysis of brucein D (BD)-induced apoptosis by PI/RNase staining assay. Cells were collected after $0,0.55,1.1$ and $2.2 \mu \mathrm{M}$ BD treatment for $32 \mathrm{~h}$, followed by PI staining and flow cytometric analysis. $M$, subG $_{1}$ phase; $P, S$ phase; $N, G_{0}+G_{1}$ phase; $Q, G_{2}+M$ phase.

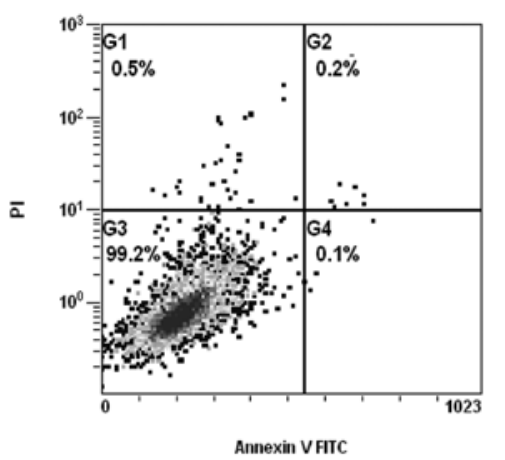

Control

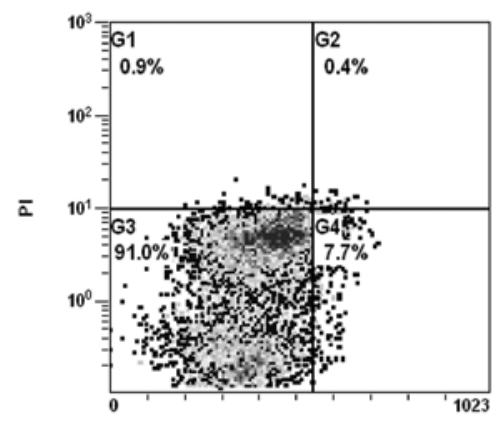

Annexin VFTC

$1.1 \mu \mathrm{M}$
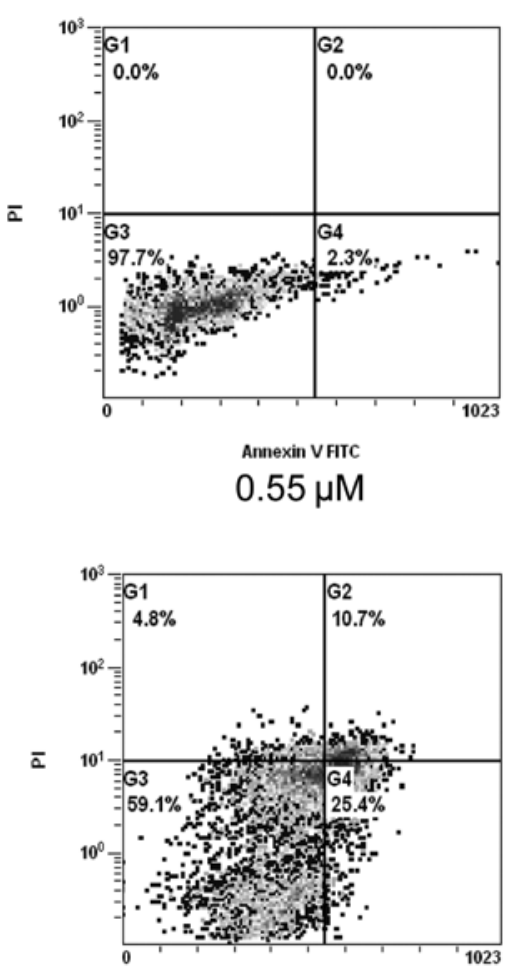

Annexin VFITC

$2.2 \mu \mathrm{M}$

Figure 5. Quantitative analysis of brucein D (BD)-induced apoptosis by Annexin V-PI staining assay. Cells treated with $0,0.55,1.1$ and $2.2 \mu \mathrm{M}$ BD treatment for $32 \mathrm{~h}$ were double stained with Annexin V and PI, followed by flow cytometric analysis. G1, G2, G3 and G4 quadrants represent necrotic, secondary necrotic, viable and apoptotic cells, respectively. 


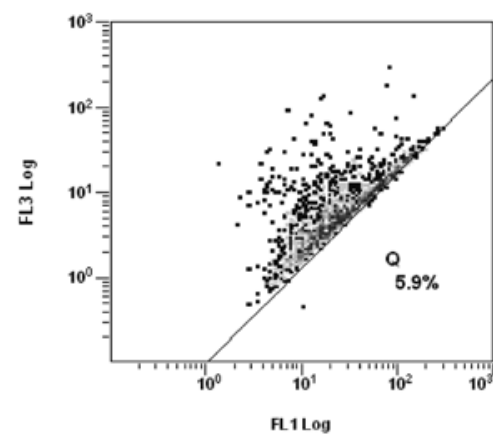

Control

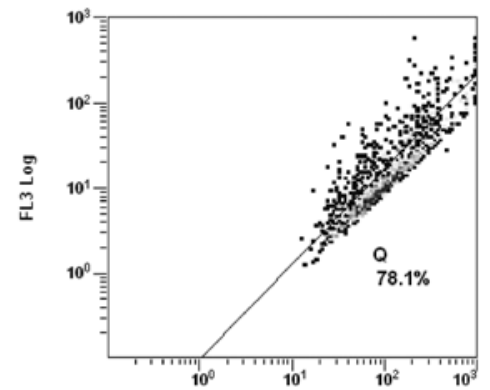

$1.1 \mu \mathrm{M}$
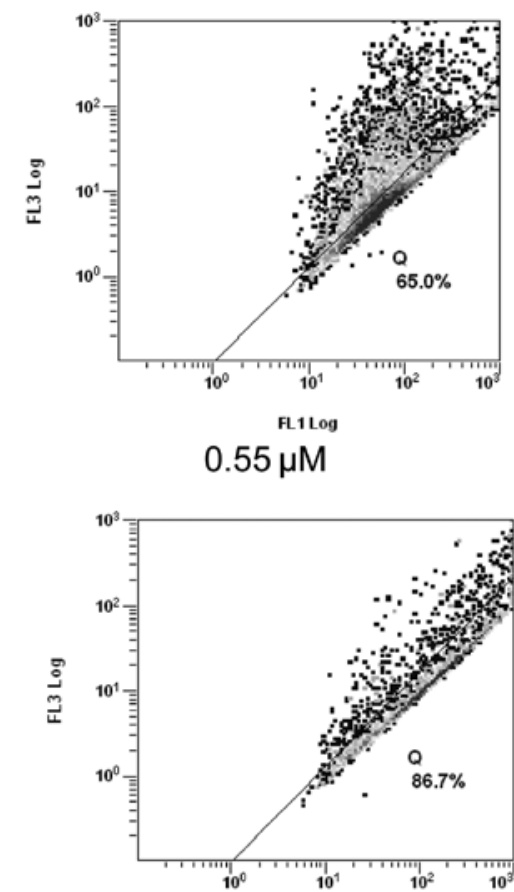

FL1 Log

$2.2 \mu \mathrm{M}$

Figure 6. Estimation of the changes of mitochondrial membrane potential on medium- and BD-treated Capan-2 cells. Capan-2 cells were incubated with BD at $0,0.55,1.1$ and $2.2 \mu \mathrm{M}$ for $24 \mathrm{~h}$. The cells were stained by JC-1 followed by flow cytometry analysis. Note that the spectral shift of the fluorescence curve to the left indicates mitochondrial membrane depolarization.

A

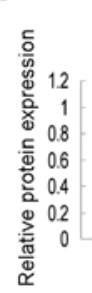

BCL-2

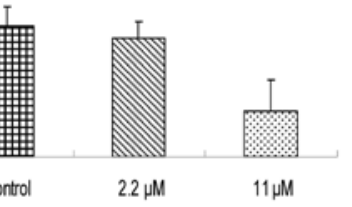

BCL-2

C

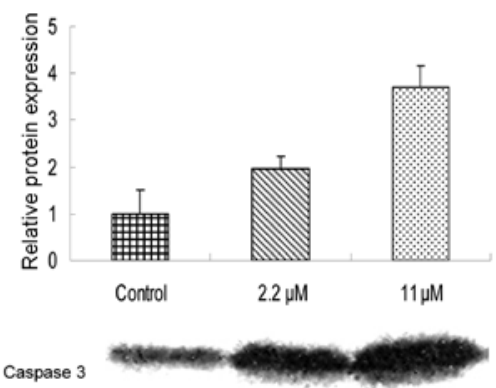

B Caspase 9

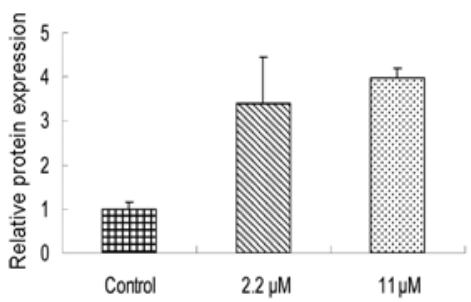

Caspase 9

D

Bactin

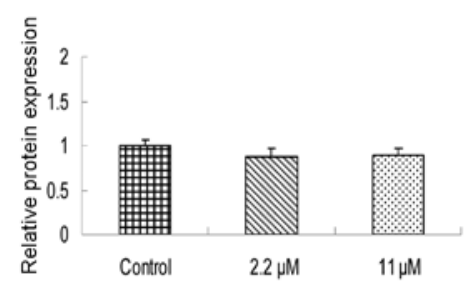

$\beta$-actin

Figure 7. Western blot analysis of the expression of apoptotic caspases in BD-treated Capan-2 cells. Treatment with BD at 2.2 and $11.0 \mu \mathrm{M}$ reduced the protein expression of Bcl-2 (A), and augmented the expression of caspase 9 (B) and caspase 3 (C). (D) $\beta$-actin was used to show that equal amounts of whole cell extracts were fractionated by SDS-PAGE.

\section{Discussion}

The fruit of Brucea javanica as a Chinese herb was first recorded in a Chinese material medical book called 'Supplementations to the Compendium of Chinese Materia Medica' by Zhao Xue-Ming some 250 years ago. One of the main functions of 
this herb is to clear heat and remove toxicity, and it is commonly prescribed by traditional Chinese medicine practitioners to treat a variety of cancers including esophageal carcinoma, small cell lung cancer, stomach cancer and pancreatic cancer (9,13-15). However, active chemical ingredients responsible for the clinical anticancer efficacy of this herb have so far not been thoroughly investigated.

Our previous studies focusing on B. javanica for pancreatic cancer have identified bruceine D (BD), a quassinoid found in relatively large quantity in the fruits of $B$. javanica, as a major active chemical ingredient possessing potent antipancreatic cancer activity. BD was able to inhibit the growth of a number of pancreatic cancer cell lines including Panc-1, SW1990 and Capan-1 cells. Panc-1 cells were established from a well-differentiated pancreatic adenocarcinoma with duct metastasis, while SW1990 cells were derived from metastasis of the spleen and Capan-1 from a well-differentiated pancreatic adenocarcinoma with liver metastasis. It is worth noting that the Capan- 2 cell line used in the present study has an origin of a primary pancreatic tumor.

It is important to examine the anti-pancreatic cancer activities of BD in various cell types of different tissue origins so that the cellular selectivity of this compound can be elucidated. The selectivity data is of significance should BD is to be developed into clinical treatment for pancreatic cancer. Our results showed that BD significantly inhibited the growth of Capan-2 cells with an $\mathrm{IC}_{50}$ value of $1.10 \mu \mathrm{M}$, whereas it only exerted modestly cytotoxic towards other non-tumorigenic cells including WRL68 and PPC. These results add further evidence to suggest that BD possesses good selectivity on pancreatic cancer cells without inflicting marked cytotoxicity to other cells of non-tumorigenic origin.

Since cellular apoptosis is a major pathway leading to cell growth inhibition, the present study elucidated whether cellular apoptosis is the underlying mechanism of action responsible for BD-induced Capan-2 growth inhibition using a variety of morphological and biochemical assays.

In mammalian cells, signalling cascades leading to apoptosis can be divided into two broad groups, the intrinsic pathway and extrinsic pathway. The intrinsic pathway, also called mitochondrial pathway, is characterized by the central role of mitochondria in the initiation of the caspase cascade executing the apoptosis program (16). As depicted in Fig. 6 , we found a dose-dependent decrease of the mitochondrial membrane potential in BD-treated Capan- 2 cells as detected by the JC-1 assay. Meanwhile, western blot analysis also showed that BD exposure attenuated the expression of $\mathrm{Bcl}-2$ protein in Capan-2 cells. In addition, in the BD-treated Capan- 2 cells both caspase 9 and caspase 3 protein expression were significantly accentuated. Moreover, BD was capable of inducing the fragmentation of genomic DNA in Capan-2 cells as evidenced by Hoechst staining. Cell cycle analysis revealed that $\mathrm{BD}$ dose-dependently increased the occurrence of subG $\mathrm{H}_{1}$ phase (apoptosis cells) Capan- 2 cells. Concomitant Annexin V and PI staining also revealed that BD was able to cause cellular apoptosis in Capan-2 cells. Taken together, our results demonstrate that the underlying mechanism of action responsible for the BD-induced cell growth inhibition on cultured Capan-2 cells is the cellular apoptosis involving the mitochondrial pathway.
In conclusion, the present study provides further evidence demonstrating that bruceine $\mathrm{D}$ is a promising anti-pancreatic cancer chemical agent. The results offer scientific support for the use of the fruit of Brucea javanica, from which BD is derived, for treatment of pancreatic cancer. Further experiments to evaluate the anti-pancreatic cancer effects of BD using an orthotopic transplantation animal model of pancreatic cancer are ongoing in our laboratory.

\section{Acknowledgements}

This project was supported by a Direct Grant from The Chinese University of Hong Kong (project no. 2030326).

\section{References}

1. Wanebo HJ and Vezeridis MP: Pancreatic carcinoma in perspective. A continuing challenge. Cancer 78: 580-591, 1996.

2. Blackstock AW, Cox AD and Tepper JE: Treatment of pancreatic cancer: current limitations, future possibilities. Oncology 10: 301-307, 1996.

3. Jemal A, Siegel R, Ward E, Hao Y, Xu J, Murray T and Thun MJ: Cancer statistic. CA Cancer J Clin 58: 71-96, 2008.

4. Oettle. H, Post S, Neuhaus P, Neuhaus P, Gellert K, Langrehr J, Ridwelski K, Schramm H, Fahlke J, Zuelke C, Burkart C, Gutberlet K, Kettner E, Schmalenberg H, Koehler KW, Bechstein WO, Niedergethmann M, Wolf IS, Roll L, Doerken B and Riess H: Adjuvant chemotherapy with gemcitabine vs observation in patient undergoing curative-intent resection of pancreatic cancer: a randomized controlled trial. JAMA 297: 267-277, 2007.

5. Barton-Burke M: Gemcitabine: a pharmacologic and clinical review. Cancer Nurs 22: 176-183, 1999.

6. Hui YF and Reitz J: Gemcitabine: a cytidine analogue active against solid tumors. Am J Health Syst Pharm 54: 162-170, 1997.

7. Chang C, Zhu YQ, Mei JJ, Liu SQ and Luo J: Involvement of mitochondrial pathway in NCTD-induced cytotoxicity in human hepG2 cells. J Exp Clin Cancer Res 29: 145, 2010.

8. Lau ST, Lin ZX, Zhao M and Leung PS: Brucea javanica fruit induces cytotoxicity and apoptosis in pancreatic adenocarcinoma cell lines. Phytotherapy Res 22: 477-486, 2008.

9. Lau ST, Lin ZX, Liao YH, Zhao M, Cheng CHK and Leung PS: Brucein D induces apoptosis in pancreatic adenocarcinoma cell line PANC-1 through the activation of p38-mitogen activated protein. Cancer Lett 281: 42-52, 2009.

10. Lin ZX, Hoult JRS and Raman A: Sulforhodamine B assay for measuring proliferation of a pigmented melanocyte cell line and its application to the evaluation of crude drugs used in the treatment of vitiligo. J Ethnopharmacol 66: 141-150, 1999.

11. Suen PM, Zou C, Zhang YA, Lau TK, Chan J, Yao KM and Leung PS: PDZ-domain containing-2 (PDZD2) is a novel factor that affects the growth and differentiation of human fetal pancreatic progenitor cells. Int J Biochem Cell Biol 40: 789-803, 2008.

12. Leung KK, Suen PM, Lau TK, Ko WH, Yao KM and Leung PS: PDZ-domain containing-2 (PDZD2) drives the maturity of human fetal pancreatic progenitor-derived islet-like cell clusters with functional responsiveness against membrane depolarization. Stem Cells Development 18: 979-990, 2009.

13. He LJ, Lou HQ and Xiang L: Efficacy of Brucea javanica oil emulsion combined radiotherapy on treating advanced esophageal carcinoma. Chin J Exp Trad Med Formul 16: 212-215, 2010.

14. Zhao BY and Fang M: Brucea javanica oil emulsion combined cisplatin and etoposide in the treatment of small cell lung cancer in advanced stage. J Clin Pulmon Med 15: 1233-1234, 2010.

15. Lu HJ, Xu H and Ning SQ: Treatment of advanced gastric cancer with DX chemotherapy and Bruceolic oil emulsion. Pract J Clin Med 7: 76-77, 2010.

16. Wang Q, Frolova AI, Purcell S, Adastra K, Schoeller E, Chi MM, Schedl T and Moley KH: Mitochondrial dysfunction and apoptosis in cumulus cells of type I diabetic mice. PLoS One 5: e15901, 2010. 